\title{
HYDROCEPHALUS
}

PROGRESSIVE HYDROCEPHALUS: SYMPTOMS AND SIGNS

The etiologies and clinical features of progressive hydrocephalus in 107 children, 56 with and 51 without shunts, were analyzed retrospectively at the Department of Neurology, Royal Hospital for Sick Children, Edinburgh, Scotland. Patients with arrested hydrocephalus, or with ventriculomegaly resulting from atrophic or ischemic brain damage or tumor were excluded. Intracranial pressure was measured percutaneously or through ventriculostomy reservoirs, using a Gaeltec miniature strain gauge transducer. Etiologies included spina bifida (54\%), idiopathic (15\%), hemorrhage (13\%), and meningitis $(10 \%)$. In those with malfunctioning shunts, symptoms were vomiting, drowsiness, headache, behavioral change, and anorexia; and signs were absent in $25 \%$ and included decreased level of consciousness in $18 \%$, acute strabismus (18\%), neck retraction (11\%), and distended retinal veins $(11 \%)$. Patients without shunts were asymptomatic in $49 \%$; headache occurred in $33 \%$, and vomiting in $16 \%$. Signs in the nonshunted group included abnormal head growth in $76 \%$, tense fontanelle (65\%), scalp vein distention (33\%), setting sun sign or absent upvard gaze $(22 \%)$, and neck rigidity (14\%). Unusual clinical features included neurogenic pulmonary edema, profuse sweating, macular rash, ptosis, autonomic dysfunction, and neurogenic stridor. Papilledema occurred in only eight cases (8\%). The authors emphasize the variability, unreliability, unusual nature, and even absence of clinical symptoms and signs of hydrocephalus with raised intracranial pressure. CT or MRI may not be diagnostic, and direct measurement of intracranial pressure is essential in patients with unexplained clinical features. (Kirkpatrick M, Engleman H, Minns RA. Symptoms and signs of progressive hydrocephalus. A.rch Dis Child Jan 1989; 64:124-128).

COMMENT. The infant referred because of a large head is a fairly common problem in pediatric neurology practice. This instructive article points out that we may be relying too frequently on our colleagues in neuroradiology for diagnostic help and neglecting the much simpler and more economical method of direct measurement of intracranial pressure. The finding that one-half the infantile cases of hydrocephalus were without symptoms is disturbing.

\section{MANAGEMENT OF HYDROCEPHALUS WITH IC PRESSURE MONITCR}

Thirteen premature infants with posthemorrhagic hydrocephalus were treated by repeated aspiration of cerebrospinal fluid using a subcutaneous ventricular catheter reservoir at the Departments of Paediatrics and Neurosurgery, University of Heidelberg, Federal Republic of Germany. Criteria for the insertion of the catheter and reservoir were as follows: 1) Increase in head circumference of more than $1 \mathrm{~cm} /$ week; 2) Progressive ventricular dilatation on ultrasound scan; 3) Failure of lumbar puncture route of fluid removal; or 4) Bradyardia or apneic complications of lumbar puncture. Hydrocephalus was controlled by aspiration of fluid (median 6ml) one to four times a day for an average of 40 days. Clinical signs (tense fontanelle and increasing head size) and ultrasound were unreliable indicators of the amount and frequency of fluid removal. Direct intracranial pressure measurements made through the reservoir increased the efficacy and 